\title{
Scaling in Wall Turbulence: Scale Separation and Interaction (Invited Paper)
}

\author{
B. J. McKeon*
}

\begin{abstract}
High Reynolds number pipe flow data are used to demonstrate the importance of several conditions related to scale separation that are either assumed in the classical theories or may be used in light of recent results in wall turbulence to infer a minimum Reynolds number condition above which scaling results may be suitable for extrapolation. Results from the Princeton Superpipe have suggested $R e_{\tau}>5000$ as the minimum Reynolds number for which key properties of pipe flow reach a "fully-developed" condition, based on observations of streamwise mean and turbulent velocity structure. Additional values related to finer constraints on the structural development are also discussed. A "skeleton" of wall turbulence is introduced, based on structural components identified as having a dominant role in the dynamics of near-wall turbulence in recent experiments by a variety of authors. Possible interaction mechanisms between these components are described alongside some outstanding questions concerning scale separation and interaction.
\end{abstract}

\section{Introduction}

The determination of the scaling behavior of smooth-wall turbulence as the Reynolds number becomes large is a question of obvious importance in both a fundamental and applied sense, and as such has consistently attracted interest from a range of researchers. However a definitive determination of flow behavior in a regime which can be successfully extrapolated to Reynolds numbers typical of aerospace applications has historically proven somewhat elusive. Even the first order question of the form of the mean velocity profile continues to generate debate ${ }^{1}$, perhaps partly because of an expanding dataset across canonical flows and partly because of increasing evidence that even an approach to the asymptotic infinite Reynolds number condition only occurs at Reynolds numbers that exceed traditional estimates, perhaps by more than an order of magnitude.

Sufficient scale separation for validity of dimensional arguments which form the foundation of many of the classical theories of turbulence presents an essential framework in which to pose the question of when the Reynolds number becomes sufficiently high for turbulence that is in some sense "fully-developed". In what follows, possible indicators for the scale separation required for a high Reynolds number flow are described and data from the Princeton/ONR Superpipe are used to demonstrate when these criteria are met in pipe flow. The primary interest lies in definitions that include more properties of flow than simply the mean velocity. No distinction is made between different types of canonical flow, in as much as all the arguments for scale separation made or cited here appear to have equally valid footing in each type of flow. The focus will be on fully-developed pipe flow with some comparisons to boundary layer results.

In what follows, spectral and structural arguments concerning scale separation are used to expand discussion of what comprises a "high Reynolds number" wall-bounded flow. The different classes of coherent structure observed in turbulent flow which become more distinct as the Reynolds number increases will be used to suggest elements of a wall turbulence "skeleton".

\section{Definitions of high Reynolds number}

Investigations over the past fifty or so years into the existence of a Reynolds-number-independent overlap region in the mean velocity can be considered to comprise a search for a high Reynolds number limit on

*Graduate Aeronautical Laboratories, California Institute of Technology (mckeon@caltech.edu). AIAA Member. 
the structure of wall turbulence. However the mean velocity is likely to be the least sensitive indicator of turbulence self-similarity, and the scaling in the overlap region continues to be a source of debate $e^{2,3,4,5}$. Here we propose and/or highlight several criteria, with an emphasis on the concept of scale separation. Note that there will be no explicit discussion of what are thought to be low Reynolds number corrections to asymptotic scaling forms, for example the forms for the overlap region in the mean velocity at low $y^{+}=y u_{\tau} / \nu$, where $y$ is the wall-normal coordinate, $u_{\tau}$ is the friction velocity and $\nu$ is the kinematic viscosity proposed by, e.g., Oberlack $^{6}$ and Buschmann \& Gad-el-Hak. ${ }^{5}$

\section{A. Self-similarity of the mean velocity profile}

1. Existence of logarithmic overlap region

Complete similarity of the mean velocity requires a minimal separation of scales, as given by

$$
\nu / u_{\tau} \ll y \ll R
$$

where $R$ is the pipe radius. Logarithmic scaling of the mean velocity, $U$, shown below in inner scaling has been observed to some degree of accuracy in many datasets over several decades.

$$
\frac{U}{u_{\tau}}=U^{+}=\frac{1}{\kappa} \ln y^{+}+B
$$

Arguments for a lack of self-similarity aside, difficulties have arisen in determining the values of $\kappa$ and $B$, the von Kármán and additive constants respectively, which have often appeared to have a Reynolds number dependence. The values of these constants have required repeated reassessment as higher Reynolds number data have become available, while Nagib et $a l^{7}$ have shown that the accuracy of skin friction measurement required to differentiate definitively between competing theories has only rarely been achieved in boundary layers.

Results from the Princeton/ONR Superpipe (see Zagarola \& Smits, ${ }^{3}$ henceforth ZS, and McKeon et $a l^{8}$ for details of the facilities, experiments and high Reynolds number probe corrections) in a Reynolds number range at present unique to this facility $\left(30 \times 10^{3}<R e_{D}=\frac{\bar{U} D}{\nu} \leq 35 \times 10^{6}\right.$, where $\bar{U}$ is the bulk or average velocity) have further enlivened the debate. A self-consistent analysis of these data (see, e.g. McKeon $^{9}$ ) suggested values of $\kappa=0.421 \pm 0.002, B=5.60 \pm 0.08$ and an additive constant in outer scaling $B^{*}=1.20 \pm 0.10$, for a logarithmic overlap region that is observed only for $600<y^{+}<0.12 R^{+}$, or $R^{+}>5000$. Note that simultaneous collapse in inner and outer variables can be simply checked in pipe flow such that inner and outer log law limits that describe a self-similar region of logarithmic scaling can be given. The values of the constants are in contrast to classical values such as $\kappa=0.41$ for $y^{+}>100$, approximately ${ }^{10}$.

\section{Ratio of outer velocity scales}

Derivation of the logarithmic law requires the use of the friction velocity as the velocity scale imposed on the outer flow from the wall boundary conditions, however ZS introduced an alternative, more truly "outer" scale, namely the difference between the velocity on the pipe centerline and the bulk (or average) velocity, i.e. $u_{Z S}=U_{C L}-\bar{U}$. ZS demonstrated that this scale can be used to obtain complete collapse of the outer region of the mean velocity profile even at low Reynolds number when only approximate collapse is obtained using the friction velocity (see also McKeon ${ }^{9}$ ). That the ratio of scales, here designated $\xi$,

$$
\xi=\frac{U_{C L}-\bar{U}}{u_{\tau}}=\frac{u_{Z S}}{u_{\tau}}
$$

tends to a constant is also a necessary condition for self-similarity as implied by logarithmic overlap ${ }^{11}$. In this regime, of course, either outer velocity scale can be used for successful scaling since the friction velocity is proportional to the $\mathrm{ZS}$ scale.

Results from the Superpipe, augmented by LDA from den Toonder \& Nieuwstadt ${ }^{12}$ in figure 1 show a distinct shape to the variation of $\xi$ with Reynolds number. A constant value is only obtained for $\operatorname{Re}_{D}>$ $300 \times 10^{3}$, although there appears to be a plateau of limited extent at lower Reynolds numbers. The transition between these two conditions is distinct and relatively abrupt in the region $70 \times 10^{3}<R e_{D}<300 \times 10^{3}$. The Kármán number corresponding to the constancy of $\xi$ is $R e_{t} a u \approx 6500$; it should be noted that very few 


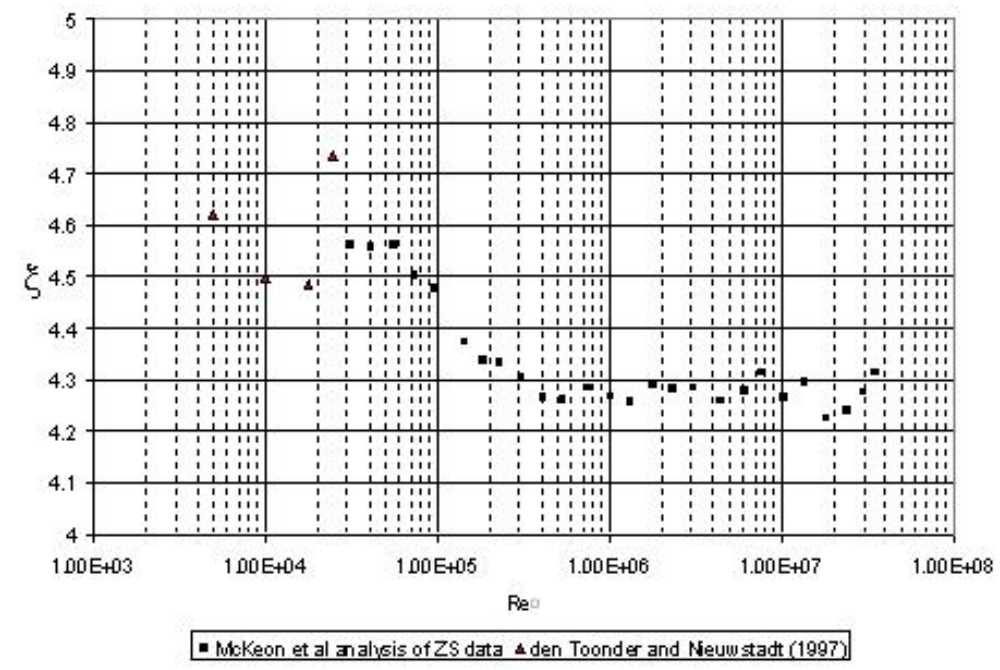

Figure 1. Variation of the ratio of outer velocity scales, $\xi$, with Reynolds number. $\square$ Superpipe data, as described in McKeon et $a l ;^{8} \wedge$ data from the LDA study of den Toonder \& Nieuwstadt. ${ }^{12}$

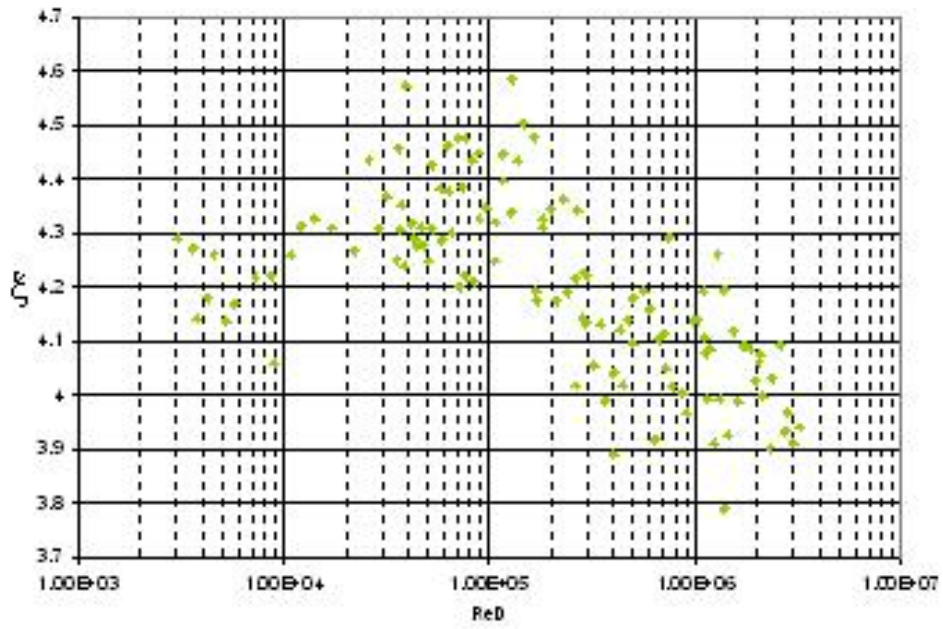

Figure 2. Variation of the ratio of outer velocity scales, $\xi$, with Reynolds number in the smooth-wall data of Nikuradse. ${ }^{13}$ 
facilities can achieve this condition and especially exceed it by a sufficient factor that scaling arguments can be made. An exception is likely to be the CICLOPE facility currently under construction in Italy. ${ }^{14}$

Similar distinct trends are also observed in the oft-maligned smooth-wall data of Nikuradse. ${ }^{13}$ While some issues with this dataset are well-known, ${ }^{15},{ }^{16}$ etc., it is still used in the formulation of Prandtl's "universal" law of friction, which yields $\kappa=0.407$. Note, however, that formulation of the friction factor law over the same high Reynolds number range as that suggested by the Superpipe results ${ }^{9}$ leads to an encouraging value of $\kappa=0.417$. Figure 2 shows the variation of $\xi$ for the Nikuradse data. While the values of the constants and the critical Reynolds numbers differ (which is consistent with the impact of differences in the wake component between this and more recent pipe flow studies, presumably a consequence of the apparatus used to generate the pipe flow) and despite large experimental scatter, the existence of a similar trend is obvious.

It is an open question as to whether this phenomenon is unique to pipe flow. It should be noted that the same trend has not been observed to date in the equivalent proportional boundary layer parameter, the Coles wake factor. While early results from Coles $^{17}$ do suggest a similar trend at high Reynolds number, the less well-defined nature of the boundary layer and large experimental scatter place doubt on the veracity of this observation.

Given this evidence (from one of the highest current laboratory Reynolds number experiments) that even the simplest test of self-similarity and separation of scales requires a Reynolds number that is high relative to what can be achieved in many laboratory facilities, we consider now scale arguments in spectral space. McKeon $e t a l^{8}$ and McKeon \& Morrison ${ }^{18}$ have shown that the distinct transition in $\xi$ accompanies several other changes in the scaling behavior of pipe flow required for a consistent definition of self-similarity of the mean velocity profile, and in terms of the turbulent fluctuations, as described below.

\section{B. Scale separation: structural self-similarity via the power spectra of turbulent fluctuations}

A more thorough test for high Reynolds number behavior involves self-similar scaling of the turbulent spectrum. There are several hypothesized spectral regimes in which this can be assessed, e.g. Perry \& $\mathrm{Li},{ }^{19}$ which are not necessarily expected to develop at the same (global) Reynolds number. Here, in light of recent debates about spectral scaling, in particular the $k_{1}^{-1}$ wavenumber scaling associated with high Reynolds number and a spectral region remote from viscous effects and in which there is no dominant imposed lengthscale, i.e.

$$
\frac{1}{R}<k_{1}<\frac{1}{y}
$$

(which has proven somewhat elusive in recent results), we present arguments in terms of the power spectrum of turbulent fluctuations, with a focus on the streamwise component.

Recent work has illuminated the growing importance of very large scale motions ${ }^{20,21}$ or super-structures ${ }^{22}$ on the near-wall region. These have been traditionally defined as having $O(10 \delta)$ extent in the streamwise direction (using Taylor's hypothesis of frozen turbulence to infer a constant convective velocity for all scales from a long temporal record). It was shown in Morrison et al ${ }^{21}$ that the growing influence of these large scales precludes $k_{1}^{-1}$ scaling, at least in the mid-to-outer reaches of the overlap region (where it has traditionally been observed from the slope of the power spectrum and without checks on simultaneous collapse). The prediction of the attached eddy hypothesis ${ }^{19}$ is still that this scaling can be observed close to the wall, e.g. Nickels et $\mathrm{al}^{23}$ who propose that $y / \delta<0.019$ and $y^{+}>100$ is required to observe this scaling in a turbulent boundary layer, an equivalent minimum Reynolds number of $R e_{\tau} \approx 5000$.

In terms of smaller scales, especially with respect to local-Reynolds-number-dependence of non-dimensional dissipation as observed in a collection of flows (e.g. Pearson et al, ${ }^{24}$ Dimotakis $^{25}$ ), Wosnik et $a l^{26}$ and McKeon \& Morrison ${ }^{18}$ have focused attention on the scale separation required for inertial scaling in the sense of Kolmogorov. The former proposed that there existed a meso-layer in inner scaling, in which the local Reynolds number is always simply too low for inertially-dominated turbulence. The conclusions of the latter are similar and are summarized here.

A search for Kolmogorov-type scaling in the overlap layer (often called the "inertial sublayer") can be justified because of approximately constant cross-flux (transport) of energy in physical space and at least approximate local equilibrium in spectral space (energy transfer), ${ }^{27}$ as shown in cartoon form in figure 3 ( $\Pi$ is the turbulent energy production, $T_{y}$ is energy transfer in physical space, mostly from the near-wall production peak to the outer flow, $T_{r}$ is energy transport in spectral space, $\epsilon$ is the local turbulent dissipation and the ordinate scale is logarithmic). Development of inertial-type scaling corresponds to spectral separation of 


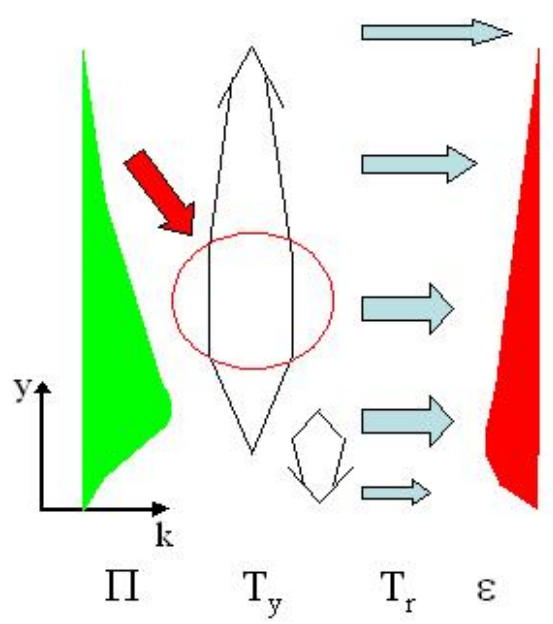

Figure 3. Cartoon of spatial and spectral energy Figure 4. Schematic of the relative variation of transfer processes in high Reynolds number wall scales relevant to the scaling of wall turbulence turbulence.

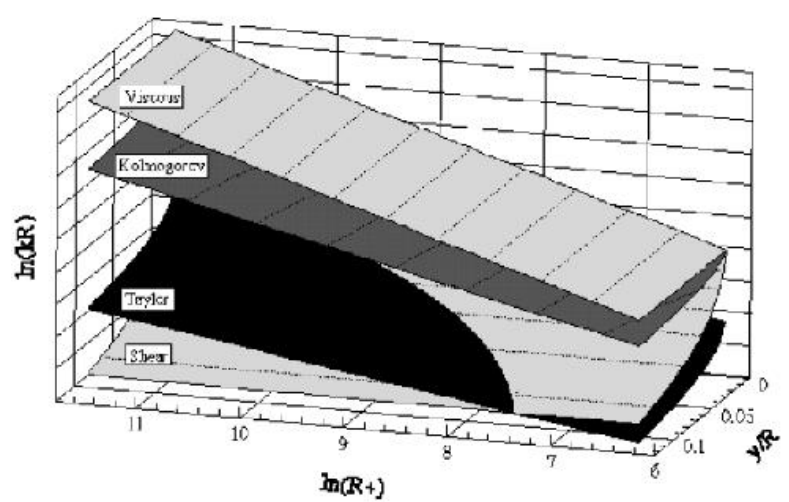

with Reynolds number in the wall region.

scales required for small-scale self-similarity of streamwise fluctuations, namely

$$
\frac{1}{y} \ll k_{1} \ll \frac{1}{\eta},
$$

where $\eta$ is the Kolmogorov lengthscale. The onset of inertial scaling is associated with a change in the shape of small-scale spectrum, most easily observed as the emergence of a "shoulder" 18 in the power spectrum premultiplied by $k^{-5 / 3}$, which develops into a plateau as the Reynolds number increases. Looking at the outer edge of overlap layer as the location in the overlap region where the local Reynolds number is highest, the approximate scale separation required for self-similarity of the streamwise fluctuations requires global Reynolds number of $R e_{\tau}>9000$, a stricter constraint than that suggested by the mean velocity results above. McKeon \& Morrison ${ }^{18}$ proposed that the requirement on relative scale separation could be extended to include the relative magnitudes of the viscous, Taylor $\left(\lambda_{T}\right)$ and shear $\left(S^{*} \sim 1 / \kappa y\right.$ in a log law region) scales as an extension of the two-dimensional schematic in Dimotakis ${ }^{25}$ to non-homogeneous shear (figure 4 , from $\left.^{18}\right)$ :

$$
\nu / u_{\tau}<\eta<\lambda_{T}<S^{*}<R .
$$

No statement can be made concerning the presence or absence of local isotropy and investigation of the small-scale spectra in the other velocity components will likely place more stringent conditions on Reynolds number. Note also that a surprising observation that similarity of the spectrum at very large scales was at least approximately achieved at the same Reynolds number indicated for small-scale similarity in the streamwise fluctuations was made, with implications for the scaling of location of large-scale energy peak (discussed below).

\section{A "skeleton" of high Reynolds number wall turbulence}

Current understanding of spatial fields in wall turbulence suggests an underlying, structural "skeleton" of turbulence, consisting of loosely-grouped structural elements that appear to be common to all canonical wall flows and which, alongside a (likely complex) dispersion relationship that is a function of scale, wall-normal location and Reynolds number, must ultimately be responsible for the scaling of temporal data described above. A brief description of these structural groups follows and a summary of known relationships between them is given below.

\section{A. Skeleton components}

A suitable discrimination between structural elements is as follows:

- Components of the near-wall ("autonomous") $\operatorname{cycle}^{28}$ 
- "Large-scale", aligned elements of the hairpin packet paradigm ${ }^{29}$ (possibly self-generating ${ }^{30}$ )

- Very-large scale streamwise meandering structures ${ }^{20}{ }^{2132}$ (a signature associated almost universally with flows exhibiting significant shear, also with non-modal growth in laminar flow).

\section{B. Scaling}

While the scaling of the near-wall cycle has been illuminated by experiments and DNS, the scaling of the other two structural groups remains something of a topic of investigation. Hairpin packets in low- to midReynolds number flows have been investigated extensively by, among others, Adrian et al, ${ }^{29}$ and uniform momentum zones associated with packets in lower Reynolds number flows have been observed in the nearneutral atmospheric layer, ${ }^{33}$ the integrity of such structures far from the wall in high Reynolds number flows is unclear ${ }^{34}$. There is evidence that hairpin structures are observed in negative large-scale velocity excursions $^{32}$ and that the turbulence intensity associated with small scales is suppressed during such verylarge-scale periods. ${ }^{22}$ There is a need for more detailed spatio-temporal information which will permit analysis of these interactions without the need to rely on Taylor's hypothesis of frozen turbulence at these very low temporal frequencies, e.g., ${ }^{1}$ which is commonly used to make the distinction between the large structural elements, $\delta$ - and $O(10 \delta)$ inferred wavelengths.

In terms of the very large scales, it has been suggested that these structures can be described in outer scaling, such that amplitude modulation of the sort shown $\mathrm{in}^{31}$ (a potential bypass mechanism for the turbulent cascade in terms of large and small scale interaction) represents an "inner-outer" interaction most obviously reflected in the continuing growth of the near-wall streamwise turbulence intensity peak with increasing Reynolds number. Hutchins \& Marusic ${ }^{22}$ have proposed that the peak value of the energy associated with the very large scale motions occurs at a constant outer-scaled wall-normal location $y / \delta$ based on boundary layer data obtained at $R e_{\tau}=1000$ and $R e_{\tau}=7300$. Given the arguments above concerning a minimum Reynolds number for reduction of finite Reynolds number effects, we use the Superpipe data to investigate the validity of inner and outer scaling of this peak in pipe flow. The premultiplied spectra $k \phi(k) / u_{\tau}^{2}$ were examined over a range of wall-normal locations and Reynolds numbers in order to determine the peak location and magnitude. Note that while the Superpipe affords a range of Reynolds numbers, the increasing size of a fixed probe relative to the viscous scale suggests that resolution effects will ultimately become important. $k_{\text {wire }} \eta \sim 0.2$ for the $R e_{\tau}=2 \times 10^{4}$ data shown below, however this is not expected to impact resolution of the large scales with the possible exception of the highest Reynolds number point. In addition, exact determination of the peak is difficult at low Reynolds number where it may overlap with the near-wall peak, and also hard at higher Reynolds number where a broad plateau of high energy exists.
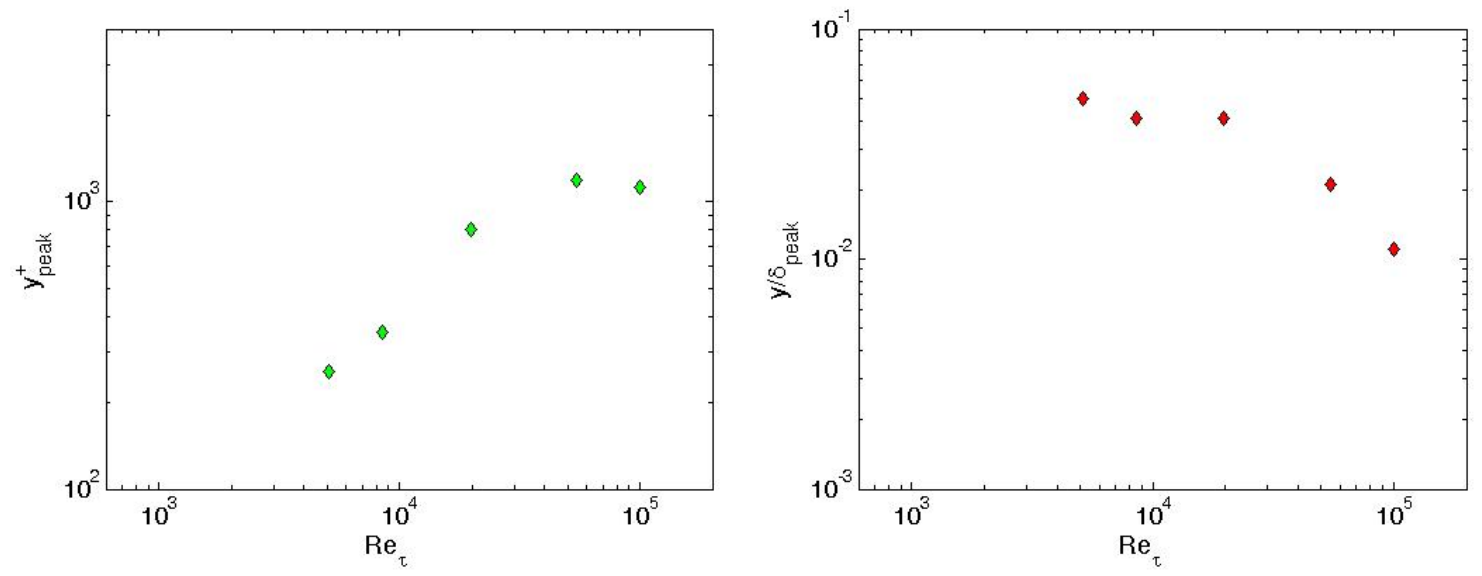

Figure 5. Location of the streamwise energy peak Figure 6. Location of the streamwise energy peak associated with the very large scale motions in associated with the very large scale motions in pipe flow in inner scaling. pipe flow in outer scaling.

The locations of the very large scale energy peak deduced from the Superpipe data reported in Morrison et $a l^{21}$ are shown in inner and outer scaling in figures 5 and 6 . The peak location does not appear to scale simply with either inner or outer variables. In addition, the peak value appears to saturate or at least 


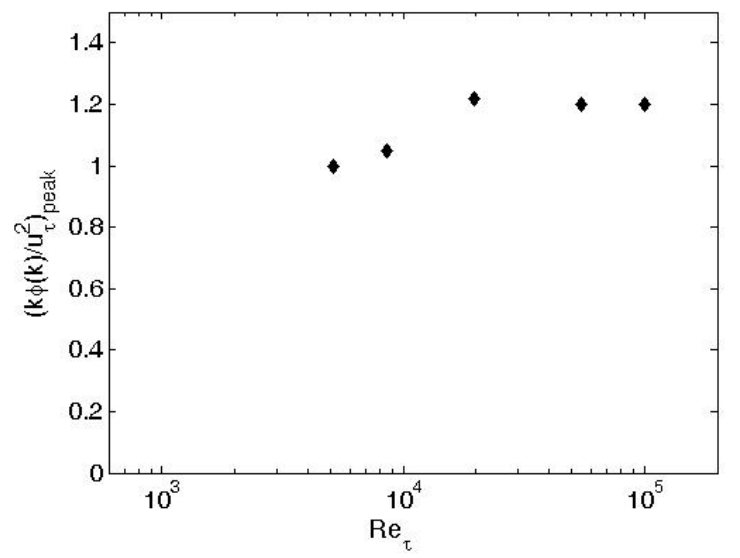

Figure 7. Variation of the magnitude of the streamwise energy peak associated with the very large scale motions in pipe flow with Reynolds number.

asymptote to a value of the order of $k \phi(k) / u_{\tau}^{2} \approx 1.2$, as shown in figure 7 (although once again resolution effects may become important). At the least, these results suggest that the nature of the scaling of the very large structures requires further investigation.

\section{Conclusions}

Several requirements on scale separation reflecting approaches to self-similarity in physical and spectral space have been discussed and compared, with a particular focus on pipe flow. It appears that $R e_{\tau}=5000$ is a good candidate for a minimum value of the Reynolds number required before the flow adopts behavior characteristic of high Reynolds number. This limit is based on observations of self-similarity of the streamwise velocity.

The concept of a "skeleton" of turbulence, likely comprising three structural groups (potentially with different origins) holds promise for modeling of wall turbulence, but requires further development. Unfortunately experiments at appropriately high Reynolds numbers are difficult. High Reynolds number still tends to imply special laboratory facilities or field campaigns, and difficulties associated with spatial and temporal resolution, and field-of-view. State-of-the-art DNS can achieve Reynolds numbers, $R e_{\tau} \sim 2000$, that are likely to remain too low to resolve these questions for the immediate future. ${ }^{35}$ Lastly we note that even the definition of "large-" and "very-large-" scale motions relies on the application of Taylor's hypothesis to large scale turbulent motions where it is not clear that the timescale can be said to be much smaller than the convective timescale. An accurate classification requires, at the least, full spatio-temporal information that yields the local dispersion relationships. It remains a challenge to understand the origin of, and relationship between, the structural groups comprising this skeleton. The relationship between the scale separation arguments made in part B of this paper and the development of the skeleton proposed in part $\mathrm{C}$ is a topic of ongoing work.

\section{Acknowledgments}

The support of the Office of Naval Research (Ron Joslin, Program Manager), for continuing investigation into scaling and scale interaction high Reynolds number flow is gratefully acknowledged. The author would like to thank Lex Smits and Jonathan Morrison for continuing use of data from the Princeton/ONR Superpipe and insightful conversations. It is a pleasure to acknowledge useful discussion with Michele Guala. 


\section{References}

${ }^{1}$ McKeon, B. J. and Sreenivasan, K. R., "Scaling and structure in high Reynolds number wall-bounded flows," Phil. Trans. Royal Soc. A, Vol. 365, 2007, pp. 635-646.

${ }^{2}$ Barenblatt, G. I., "Scaling laws for fully developed turbulent shear flows, Part I. Basic hypotheses and analysis," J. Fluid Mech., Vol. 248, 1993, pp. 516-520.

${ }^{3}$ Zagarola, M. V. and Smits, A. J., "Mean-flow scaling of turbulent pipe flow," J. Fluid Mech., Vol. 373, 1998, pp. 33-79.

${ }^{4}$ George, W. K. and Castillo, L., "Zero-pressure-gradient turbulent boundary layer," Appl. Mech. Rev., Vol. 50, 1997, pp. 689-729.

${ }^{5}$ Buschmann, M. H. and Gad-el Hak, M., "Generalized logarithmic law and its consequences," AIAA J., Vol. 41, 2003, pp. $40-48$.

${ }^{6}$ Oberlack, M., "A unified approach for symmetries in plane parallel turbulent shear flows," J. Fluid Mech., Vol. 427, 2001, pp. 299-328.

${ }^{7}$ Nagib, H. M., Chauhan, K. A., and Monkewitz, P. A., "Approach to an asymptotic state for zero pressure gradient turbulent boundary layers," Phil. Trans. Royal Soc. A, Vol. 365, 2007, pp. 755-770.

${ }^{8}$ McKeon, B. J., Li, J., Jiang, W., Morrison, J. F., and Smits, A. J., "Further observations on the mean velocity distribution in fully developed pipe flow," J. Fluid Mech., Vol. 501, 2004, pp. 135-147.

${ }^{9}$ McKeon, B. J., High Reynolds number turbulent pipe flow, Ph.D., Princeton University, Princeton, NJ, U.S.A., June 2003.

${ }^{10}$ Bradshaw, P., Topics in Applied Physics, Turbulence, Springer-Verlag, 1976.

${ }^{11}$ Yaglom, A. M., "Similarity laws for constant-pressure and pressure-gradient turbulent wall flows," Ann. Rev. Fluid Mech., Vol. 11, 1979, pp. 505-540.

12 den Toonder, J. M. J. and Nieuwstadt, F. T. M., "Reynolds number effects in a turbulent pipe flow for low to moderate Re," Phys. Fluids, Vol. 9, 1997, pp. 3398-3409.

${ }^{13}$ Nikuradse, J., "Laws of turbulent flow in smooth pipes (English translation)," NASA TT F-10.

${ }^{14}$ Talamelli, A., Persiani, F., Fransson, H. M., Alfredsson, P. H., Johansson, A. V., Nagib, H., Ruedi, J.-D., Sreenivasan, K. R., and Monkewitz, P., "CICLOPE - a response to the need for high Reynolds number experiments," Fluid Dyn. Res., in press 2008.

${ }^{15}$ Zagarola, M. V., Mean-flow scaling of turbulent pipe flow, Ph.D. thesis, Dept. of Mechanical \& Aerospace Engineering, Princeton University, Princeton, U.S.A., 1996.

${ }^{16}$ Beattie, D. R. H., "In defence of Nikuradse," Twelfth Australasian Fluid Mechanics Conference, 1995.

${ }^{17}$ Coles, D. E., "The turbulent boundary layer in a compressible fluid. Appendix A: A manual of experimental boundarylayer practice for low-speed flow." Report R-403-PR, Rand Corporation, 1962.

${ }^{18}$ McKeon, B. J. and Morrison, J. F., "Asymptotic scaling in turbulent pipe flow," Phil. Trans. Royal Soc. A, Vol. 365, 2007, pp. 771-787.

${ }^{19}$ Perry, A. E. and Li, J. D., "Experimental support for the attached-eddy hypothesis in zero-pressure-gradient turbulent boundary layers," J. Fluid Mech., Vol. 218, 1990, pp. 405-438.

${ }^{20}$ Kim, K. C. and Adrian, R. J., "Very large-scale motion in the outer layer," Phys. Fluids, Vol. 11, 1999, pp. 417-422.

${ }^{21}$ Morrison, J. F., Jiang, W., McKeon, B. J., and Smits, A. J., "Scaling of the streamwise velocity component in turbulent pipe flow," J. Fluid Mech., Vol. 508, 2004, pp. 99-131.

${ }^{22}$ Hutchins, N. and Marusic, I., "Large-scale influences in near-wall turbulence," Phil. Trans. Royal Soc. A, Vol. 365, 2007, pp. $647-664$.

${ }^{23}$ Nickels, T. B., Marusic, I., Hafez, S., and Chong, M. S., "Evidence of the $k_{1}^{-1}$ law in a high-Reynolds-number turbulent boundary layer," Phys. Rev. Letters, Vol. 95, No. 074501, 2005.

${ }^{24}$ Pearson, B. R., Krogstad, P.-Å., and van de Water, W., "Measurements of the turbulent energy dissipation rate," Phys. Fluids, Vol. 14, No. 3, 2002, pp. 1288-1290.

${ }^{25}$ Dimotakis, P. E., "The mixing transition in turbulent flows," J. Fluid Mech., Vol. 409, 2000, pp. 69-98.

${ }^{26}$ Wosnik, M., Castillo, L., and George, W. K., "A theory for turbulent pipe and channel flows," J. Fluid Mech., Vol. 421, 2000, pp. 115-145.

${ }^{27}$ Marati, N., C. C. M. . P. R., "Energy cascade and spatial fluxes in wall turbulence." J. Fluid Mech., Vol. 521, 2004, pp. 191-215.

${ }^{28}$ Jimenez, J. and Pinelli, A., "The autonomous cycle of near-wall turbulence," J. Fluid Mech., Vol. 389, 1999, pp. 335-359.

${ }^{29}$ Adrian, R. J. and Meinhart, C. D. amd Tomkins, C. D., "Vortex organization in the outer region of the turbulent boundary layer," J. Fluid Mech., Vol. 422, 2000, pp. 1-54.

${ }^{30}$ Zhou, J., Adrian, R. J., Balachandar, S., and Kendall, T. M., "Mechanisms for generating coherent packets of hairpin vortices in channel flow," J. Fluid Mech., Vol. 387, No. 353-396, 1999.

${ }^{31}$ Hutchins, N. and Marusic, I., "Evidence of very long meandering features in the logarithmic region of turbulent boundary layers," J. Fluid Mech., Vol. 579, 2007, pp. 1-28.

${ }^{32}$ Ganapathisubramani, B., Longmire, E. K., and Marusic, I., "Characteristics of vortex packets in turbulent boundary layers," J. Fluid Mech., Vol. 478, 2003, pp. 35-46.

${ }^{33}$ Morris, S. C., Stolpa, S. R., Slaboch, P. E., and Klewicki, J., "Near-surface particle image velocimetry measurements in a transitionally rough-wall atmospheric boundary layer," J. Fluid Mech., Vol. 580, 2007, pp. 319-338.

${ }^{34}$ Adrian, R. J., "Vortex organization in wall turbulence," Phys. Fluids, Vol. 19, No. 041301, 2007.

${ }^{35}$ Jimenez, J. and Moser, R., "What are we learning from simulating wall turbulence?" Phil. Trans. Royal Soc. A, Vol. 365, 2007, pp. $715-732$. 